\section{Risk of geographic atrophy in age-related macular degeneration patients treated with intravitreal anti-VEGF agents}

\begin{abstract}
Anti-vascular endothelial growth factor (VEGF) intravitreal agents are the only successful treatment for wet age-related macular degeneration (AMD). However, there are emerging signals that anti-VEGF treatment can potentially increase development of geographic atrophy (GA). Histopathologic, animal, and clinical studies support this hypothesis although direct proof of a relationship between GA and use of anti-VEGF agents in neovascular AMD is not yet established. This review presents current evidence supporting an association between anti-VEGF therapy and progression of geographic atrophy. The need of exploring alternative methods of treating AMD is indirectly but clearly emphasized.

Eye (2017) 31, 1-9; doi:10.1038/eye.2016.208; published online 7 October 2016
\end{abstract}

\section{Introduction}

Geographic atrophy (GA) can develop from both the wet and the dry forms of age-related macular degeneration (AMD). ${ }^{1}$ Retrospective data analysed from multicentre clinical trials demonstrate the necessity of long term followup of patients treated with anti-vascular endothelial growth factor (anti-VEGF) agents, both to monitor long-term visual outcomes and also to evaluate safety of this therapy with respect to the risk of developing GA. ${ }^{2-4}$

Recently, phase III clinical trials have begun to evaluate treatment for geographic atrophy (the parallel CHROMA and SPECTRI studies, ClinicalTrials.gov Identifier: NCT02247479 and NCT02247531, respectively).

Genetic predisposition to developing AMD is well-established;, 5 ,6 thus, identifying potential
M Gemenetzi ${ }^{1}$, AJ Lotery ${ }^{2,3}$ and PJ Patel ${ }^{1}$

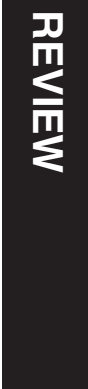

novel treatment pathways such as complement inhibition as future treatment options. Currently though, anti-VEGF therapy is the only option available to delay progression in patients affected with choroidal neovascularization (CNV). ${ }^{7}$

The anti-angiogenic approach to treating neovascular AMD (nAMD) has been undoubtedly successful but is AMD treatment at risk of becoming monolithic? Possibly, if its scientific approach is limited to causing blood vessels to regress or become less permeable. ${ }^{8}$

Geographic atrophy and CNV: pathophysiologic considerations

Before the anti-VEGF era

In 1999, before anti-VEGF treatment for nAMD, Green noted retinal pigment epithelial (RPE) atrophy in $37 \%$ of 760 eyes with AMD in a histopathologic study. ${ }^{9}$ This was associated with disciform scars. Thus, suggesting that GA is associated with CNV.

Sunness et al ${ }^{10}$ prospectively looked at the natural history of 152 patients with GA and no $\mathrm{CNV}$ by fluorescein angiography in at least one eye, with annual follow-up for 4 years. She found CNV did not develop in areas of GA, but rather in areas of preserved retina surrounding the GA or in spared foveal regions. Schatz and McDonald ${ }^{11}$ reported that $\mathrm{CNV}$ did not develop in areas of GA, when the choriocapillaris (CC) was absent as a consequence of atrophy of the RPE, which was additionally supported by previous histopathologic work. ${ }^{12}$

Sarks et $a l^{13}$ tried to trace the evolution of GA based on clinical documentation and by clinicomorphological correlation in representative eyes. They observed that 'new vessel ingrowth is
${ }^{1}$ NIHR Biomedical Research Centre for Ophthalmology, Moorfields Eye Hospital NHS Foundation Trust, London, UK

${ }^{2}$ Division of Neurosciences, Faculty of Medicine, University of Southampton, Southampton, UK

${ }^{3}$ Eye Unit, Southampton University Hospital NHS Foundation Trust, Southampton, UK

Correspondence: PJ Patel, NIHR Biomedical Research Centre for Ophthalmology, Moorfields Eye Hospital NHS Foundation Trust, London, UK

Tel: +02075662108;

Fax: +02076086925.

E-mail: praveen.patel@ moorfields.nhs.uk

Received: 23 May 2016 20 August 2016 Published online:

7 October 2016 Accepted in revised form: 
dependent upon a viable RPE and can only occur outside the area of atrophy, which limits neovascular response, so that the latter may even remain subclinical'.

Hence, there is evidence to suggest that in cases where GA precedes CNV development, the latter does not develop within the area of GA. It is therefore unlikely to 'miss' pre-existing GA in eyes with $\mathrm{CNV}$ about to be treated with anti-VEGF, especially given the availability of current advanced imaging techniques, as a result of 'masked' GA by the co-existing CNV lesion, unless there is a great amount of haemorrhages and exudates.

\section{In the anti-VEGF era}

Saint-Geniez et $a l^{14}$ found that in mice, the absence of diffusible VEGF isoforms, 120 and 164, led to an age-dependent degeneration of the RPE-CC similar to dry-atrophic AMD: beginning at 7 months of age, mice that only produced VEGF188 exhibited a progressive degeneration of the RPE/CC/Bruch's membrane, and the subsequent increased photoreceptor apoptosis led to a marked decline in visual acuity detected by electroretinography. Increased autofluorescence (AF) and accumulation of basal laminar deposits were observed, which finally evolved to focal choroidal atrophy and RPE attenuation similar to human GA. The authors also showed that there was an autocrine VEGF function in vivo and that this was necessary for the maintenance of the RPE-complex integrity. It is of note that absence of the VEGF isoforms mentioned above had an impact on the integrity of the RPE/CC complex only in older mice and was age-dependent.

'RPE atrophy ws choroidal atrophy' in AMD According to Bhutto and Lutty ${ }^{15}$ there is a 'mutualistic symbiotic relationship' between the components of the photoreceptor/RPE/Bruch's membrane/CC complex and subsequently between degenerating RPE and CC. Lutty's lab team had previously shown that at least in advanced dry AMD (GA), RPE atrophy occurs first, followed by CC degeneration, whereas CC degeneration precedes RPE atrophy in wet AMD. ${ }^{16}$

There is contradicting evidence suggesting age-related thinning of the choroid, and there are also questions regarding accuracy of measuring choroidal thickness in a clinical setting using spectral domain OCT. ${ }^{17,18}$ McLeod et $a l^{19}$ developed an image analysis methodology to quantify changes in RPE and CC in post-mortem human eyes with AMD: choroidal tissue was incubated for alkaline phosphatase activity (APase) that indicates endothelial cell viability and is found in viable choroidal blood vessels. Loss of RPE and CC was quantified using illumination for capturing images, and Adobe Photoshop used to determine the number of blue pixels from APase-stained choroidal blood vessels. ${ }^{20}$ Their technique showed that loss of RPE was related to loss of $\mathrm{CC}$ and that there is a linear relationship between the loss of RPE and loss of CC in GA.

Bhutto et al recently published their results on a study of human donor eyes on choroidal tissue of patients with clinically diagnosed AMD and choroidal tissue of agematched controls without evidence of macular disease. This was the first report to show that mast cell (MC) numbers and activation were increased in all forms of AMD, including early AMD. ${ }^{21}$ The authors stated that MCs within choroidal neovascular membranes release proteolytic enzymes, which may lead to thinning of the choroid in AMD and degradation of Bruch's membrane; this may result in RPE death and CC degeneration, which the same authors had documented before in both GA and $\mathrm{nAMD}$.

Mullins et al performed a series of morphometric experiments in which they assessed the relationship of the vasculature with sub-RPE deposits in the human macula. They found the density of pathologic deposits was strongly linked to the density of the choroidal vasculature, with eyes having the most drusen showing the lowest vascular density. ${ }^{22}$

Kaszubski et al ${ }^{1}$ very recently reviewed the literature on the epidemiology, clinical presentation, and treatment options for patients with the combined GA/CNV entity. Most of the clinical studies, mentioned in their review article, focused on the incidence of CNV in eyes with GA at baseline as in the Macular Photocoagulation Study (MPS) and the Beaver Dam Eye Study. ${ }^{23-25}$ In addition, Grob et $a l^{26}$ found that GA tends to occur before CNV development in the combined form in their study, in which they found no significant higher frequency of certain gene alleles related to specific AMD phenotypes.

In large, clinical, prospective studies described in more detail below, the appearance of the area of GA detected using colour fundus photography in patients with $\mathrm{CNV}$ and on anti-VEGF treatment at 2-year follow-up, was clinically indistinguishable from areas of GA where no CNV seemed to be present. This is consistent with the finding that photoreceptor and RPE degenerate in a horse-shoe-shaped pattern surrounding the fovea as mentioned above, ${ }^{15}$ regardless of the timing of events (does RPE atrophy occur first or does CC degeneration precede RPE atrophy?) and of the form of AMD (dry AMD or combined CNV and GA).

Evidence based on the above laboratory and clinical studies still generate questions about the molecular pathways involved in the development of the two forms of AMD, 'exudative' and 'dry', as well as about the 'combined form of GA and $\mathrm{CNV}^{\prime}$. Is it worth thinking of AMD as one disease or is it two different independently working mechanisms leading to two clinically separate 
forms? Are the molecular pathways involved in the development of geographic atrophy similar to the ones involved in the development of GA on a background of neovascularization, especially when the latter is treated with anti-VEGF?

\section{Clinical evidence on GA and anti-VEGF treatment}

\section{Prospective studies}

Comparison of AMD treatment trials Assessing the risk of geographic atrophy in the comparison of AMD treatments trials (CATT), Grunwald et al assessed lesions developing during 2 years of anti-VEGF therapy based on coloured fundus photography and fundus fluorescein angiography (FFA). ${ }^{2}$ Trained and certified graders at the CATT Fundus Photograph Reading Centre reviewed the images at baseline and at follow-ups. OCT scans were not used to assess presence of GA. Only patients without evidence of GA in the study eye at enrolment were considered as being at risk of developing incident GA (GA not present before treatment initiation).

Pertinent findings: One hundred and eighty seven (18\%) of the 1024 patients who were included in the trial developed GA by 2 years, and independent baseline risk factors associated with higher risk of GA were the following: poor visual acuity at baseline in the study eye and in the fellow eye $(P<0.03)$; retinal angiomatous proliferation (RAP; $P<0.0001$ ); presence of intraretinal fluid $(P<0.0001)$ and absence of subretinal fluid $(P<0.0001)$; monthly dosing as opposed to eyes treated PRN ( adjusted hazard ratio, $1.59 ; 95 \%$ confidence interval, $1.17-2.16$ on multivariate analysis); and treatment with ranibizumab as opposed to bevacizumab (adjusted hazard ratio, 1.43; 95\% confidence interval, 1.06-1.93). Interestingly, eyes with any subretinal fluid in the foveal centre were at a less risk than eyes without subretinal fluid.

The same group published their results on the evaluation of growth of GA in the CATT trial patients during anti-VEGF treatment. ${ }^{3}$ The CATT cohort consisted of 1185 patients with AMD-related CNV, but patients with foveal centre GA were excluded. Morphologic features of the study eyes were evaluated as in the previous study, and whereas GA detected at baseline was considered as 'prevalent GA', GA at years 1 or 2 or both was considered as 'incident $\mathrm{GA}^{\prime}$. When prevalent and incident GA were considered together, ranibizumab treatment $(P=0.02)$, GA in the fellow eye $(P=0.02)$, and area of $\mathrm{GA}$ at baseline $(P<0.001)$ were significantly associated with faster growth.

To alleviate concerns about the dependence of growth rate on initial area, the investigators included the initial area in the model of growth measurement and found that this was not associated with the growth rate. They also found no significant difference in the mean growth rate between PRN (as needed) and monthly treatment, and consequently found no significant association of the number of injections with GA growth rate. The greater the distance of the GA lesion to the fovea was the higher the growth rate of the former $(P=0.03)$. GA growth rate doubled in CNV with a classic component and it was also higher when GA developed within or in close proximity to the CNV lesion. In the group where patients switched from monthly treatment during year 1 to PRN treatment during year 2, incidence of GA was lower in year 2 .

The results of following up 529 CATT participants for 5 years to evaluate the size and growth of GA were announced at the ARVO meeting this year: GA size increased over time by a mean of $0.29(0.02) \mathrm{mm} /$ year although GA growth rate decreased from years 1 to 5 . Eyes with predominantly classic lesions and those without sub-RPE fluid at baseline exhibited higher GA growth. There was no significant difference in GA growth between GA associated with the CNV lesion and GA that was outside the CNV lesion. There was no significant difference between the two drug types either. The dosing regimen did not affect outcomes regarding GA growth at the end of the 5 -year follow-up. ${ }^{27}$

\section{Alternative Treatments to Inhibit VEGF in Age-related} Choroidal Neovascularization trial In the 2-year findings of the Alternative Treatments to Inhibit VEGF in Age-related Choroidal Neovascularization trial (IVAN), the percentage of participants with new GA was not different between drug groups but it was significantly lower in the arms where discontinuous treatment was applied $(P=0.03){ }^{4}$

In the same report it was noted that continuous treatment (monthly) offered a slightly better visual function than PRN treatment but 'this was not reflected in the primary outcome of best-corrected visual acuity or in self-reported health-related quality of life'.

Both the CATT and the IVAN trials are well-designed, prospective studies using a standardized, well-defined protocol to administer treatment and in which detection of GA was on the basis of colour fundus photography. The relationship between the development of GA and frequency of anti-VEGF treatment has been shown significant in both studies during the first 2 years of follow-up. However, the type of anti-VEGF treatment does not seem to have had an impact on the development of GA in the IVAN trial as it was in the CATT trial during the first 2 years of follow-up. The impact of anti-VEGF type was not significant in the CATT cohort of patients who were followed up for 5 years as well. The outcomes of the 5-year follow-up of this cohort of CATT patients are overall consistent with the ones of the shorter follow up of 
2 years: classic or predominantly classic CNV appears to be closely related to GA development and growth rate of GA both in the short-term as well as in the long-term follow-up. There was no significant association of the number of injections with GA growth rate in the 2-year and 5-year follow-up although the difference was significant in year 1 . It seems, perhaps frequency of treatment affects development of incident GA initially, but it has no impact on growth rate in the long term. There was one more discrepancy between the results in the 2-year and the 5-year follow-up: localization of GA in the 5-year follow-up made no difference in terms of GA growth rate and proximity to the CNV lesion as opposed to the 2-year findings, where GA growth rate was higher when closer to the CNV lesion.

It is of note that patients in the 5-year follow-up cohort had been released from protocol treatment at 2 years and presumably anti-VEGF treatment was less uniform in terms of the frequency or the type of anti-VEGF agent they were receiving after their release from the CATT study protocol.

High-contrast, distance visual acuity testing is not the only method to detect functional impairment. Other aspects of visual function such as low luminance vision, contrast sensitivity, retinal sensitivity testing (using micro-perimetry), or reading ability may be alternative ways of detecting vision function impairment in GA. These alternative tests may be more reliable than visual acuity testing, in detecting and quantifying the magnitude of visual function impairment due to GA. Indeed, improvement in visual acuity is not synonymous with improved reading ability, as Sarks et $a l^{13}$ noted several years ago, and visual retraining may direct patients to use a more 'suitable' area of retina in order to deal with loss of fixation. ${ }^{28}$ This may also vary among patients and there is no objective prognostic indicator to predict the speed or severity of disability due to vision loss in patients with GA. In part, this is due to variability of the location of GA in the macula between eyes and indeed the direction of expansion of these areas of atrophy.

One important consideration that merits emphasizing is that there is no single, gold standard imaging modality with respect to detection of GA. It is also true that in the trials discussed above, colour fundus photography was the diagnostic tool used in the assessment, which some consider the least sensitive method for the detection of GA.

However, in addition to large clinical trials, there are retrospective, 'real-world' studies with relatively large samples and a longer follow-up time where the impact of anti-VEGF treatment on the development of GA has been evaluated. Advanced imaging technology was used in most of these studies to assess the existence and progression of GA.

\section{Retrospective studies}

Gillies et al analysed the long-term outcomes of anti-VEGF treatment in 1212 eyes with nAMD in an observational study with a mean follow-up of 53.5 months. Loss of $>10$ letters occurred in $32 \%$ of the eyes that continued treatment for $>6.5$ years, and GA at the centre of the fovea was the most common cause of visual loss, accounting for $37 \%$ of the total. ${ }^{29}$ Comparing their methods and results with similar reports such as the SEVEN-UP and the UK EMR Users Group studies, 30,31 they stated more injections were given in their study and better VA results were achieved. The percentage of atrophy involving the foveal centre in the Gillies study was much lower than that recorded in others, for example, in the SEVEN-UP study. However, the authors admit, that the percentage of GA they reported as the major cause of visual loss in their study, was an underestimate, because a lot of patients who developed central macular atrophy (MA) before 6.5 years, had discontinued treatment. A little less than $10 \%$ discontinued during the first 2 years, increasing to $46 \%$ from the third to the fifth year after commencing treatment. They also accept that there were different treatment protocols, and baseline VA differed among studies so that a precise and valid comparison of all variables and results cannot be made.

Notably, in the SEVEN-UP study, although better VA outcomes were obtained in patients receiving a higher number of injections, macular thinning (atrophy) was the key anatomic determinant of long-term visual outcomes and the only variable demonstrating a significant association with final vision loss. The SEVEN-UP study was a multi-centre, cross-sectional study of the long-term outcomes of a cohort of patients treated with ranibizumab within the ANCHOR and the MARINA trials, and subsequently enrolled and treated with ranibizumab in the HORIZON study. ${ }^{32-35}$ The aim was to evaluate results after 7 years of treatment with ranibizumab. Fibrotic scars and continuous leakage were finally displayed in retinal imaging in one-third and in half of the eyes included in the study, respectively, but virtually all eyes had shown MA.

In the most recent publication of the SEVEN-UP study, MA was less severe in the study eyes than in fellow eyes with nAMD. The authors therefore concluded that monthly ranibizumab injections did not lead to atrophy progression over time. ${ }^{36}$ However, the small cohort, increased selection bias and heterogeneity of patients regarding their condition at baseline, and how they were treated over a course of 7-8 years make it difficult to draw conclusions. The assertion that long-term anti-VEGF therapy does not affect development or growth rate of GA cannot therefore be confirmed. 
Similar results and conclusions were presented in publications by the HARBOR study group: in the HARBOR trial, investigators evaluated the efficacy and safety of intravitreal ranibizumab 0.5 and $2.0 \mathrm{mg}$ administered monthly and on an as-needed (PRN) basis in treatment-naive patients with subfoveal nAMD (wet AMD). The HARBOR investigators retrospectively used coloured fundus photography and FFA to assess GA, and their results were comparable to the ones of the CATT and the IVAN trials. ${ }^{37}$

Smaller-scale retrospective studies Lois et al detected RPE atrophy using short-wavelength AF and near-infrared AF (NIA) in a retrospective review of AMD patients with CNV treated with anti-VEGF. ${ }^{38}$ They looked at atrophy at baseline and at progression of atrophy at follow-up of 72 eyes, treated with ranibizumab only, for a median of 16 months. They defined atrophy at baseline as a reduced signal in both AF and NIA of $>0.05 \mathrm{~mm} 2$ in the absence of haemorrhage, exudates, or blockage of the AF/NIA signal related to the CNV when this was subretinal. They defined progression of atrophy as any enlargement of preexisting atrophy or new atrophy as shown on both the AF and NIA images. As in most retrospective studies, there were no strict re-treatment criteria but patients were mostly treated with monthly injections until VA did not further improve in two consecutive visits. From that point onwards there were monthly follow-ups and treatment was offered on a PRN basis. Most of their patients had occult CNV. In $62 \%$ of the eyes studied, there was progression of atrophy at the last follow-up, and in 58\%, the area of atrophy involved the centre of the macula. In $84 \%$ of the eyes in which there was progression of atrophy, no atrophy was detected at baseline. The number of ranibizumab injections was significantly associated with progression of atrophy but there was no evidence that the presence of atrophy at baseline or that the length of follow-up were associated with atrophy progression. VA decreased by 0.064 logarithm of the minimum angle of resolution in eyes that had developed central atrophy at follow-up while VA increased by 0.006 in eyes without central atrophy.

Another retrospective review of patients' records seen by the retina service at the University of British Columbia included 415 eyes with nAMD treated with either bevacizumab or ranibizumab and a mean follow-up period of 2.2 years. ${ }^{39}$ Patients were treated based on a 'treat and extend' regimen. They used non-treated fellow eyes with non-nAMD as controls. In this study, Cirrus HD-OCT was used to evaluate RPE atrophy using the advanced RPE analysis tool on the Cirrus HD-OCT software. They also measured subfoveal choroidal thickness using the same software and the manual caliper function at baseline and at the final follow-up. RPE atrophy progression was significantly higher in eyes with nAMD treated with anti-VEGF than in controls $(P<0.001)$. The amount of atrophy progression was significantly and independently associated with age $(P=0.004)$, the number of bevacizumab injections $(P<0.001)$, and the number of ranibizumab injections $(P=0.001)$. The difference between atrophy and number of injections for the two types of anti-VEGF was not statistically significant. Choroidal atrophy was also independently associated with the number of anti-VEGF injections regardless of the anti-VEGF drug used, and it was more pronounced in eyes treated with anti-VEGF therapy for $n A M D$ than in controls $(P<0.001)$.

$\mathrm{Xu}$ et al used both NIA/AF and SD-OCT (spectral domain-OCT) to detect GA in nAMD patients treated with either ranibizumab or bevacizumab and/or aflibercept in a treat and extend regimen, as well as FFA to classify neovascular lesion subtypes. ${ }^{40}$ They included 94 eyes of 91 patients and a minimum of 12 months follow-up. Central GA at baseline was an exclusion criterion. Multiple logistic regression and multiple linear regression analysis were used to model odds of developing GA and to identify factors that affected a change in the area of GA. About $37 \%$ of the eyes included and that did not have apparent baseline GA, developed GA at the last follow-up, and all of the eyes that had GA at baseline $(18 \%)$ had enlargement of the GA areas. No other variables except for the number of anti-VEGF injections $(P=0.02)$ and the neovascularization type $(P<0.001)$ were related to GA development. What the authors stated as novel in this study was the combination of NIA/AF and SD-OCT to distinguish causes of hyperreflectance on NIA. They also pointed out that anatomical classification is important in the prognosis of GA risk development in eyes treated with anti-VEGF therapy for nAMD because eyes with type I CNV (occult) were previously found to be resistant to the development of GA. ${ }^{41}$ This finding is supported by Grossniklaus and Green $^{42}$ who suggested that the RPE and the photoreceptors get some nutritional support from the neovascular tissue underneath the RPE in type I neovascularization. This maybe one of the reasons that in clinical trials testing efficacy and safety of anti-VEGF therapy, visual improvement was not as significant in CNV type I as in classic CNV. ${ }^{34}$

Classification of CNV, as 'classic/well defined' or 'occult/poor defined', was initially based on FFA and described in the MPS. ${ }^{43,44}$ The idea to relate the location of the membrane with respect to the RPE belongs to Gass. He introduced the terms type I CNV to describe new vessels developing in the sub-RPE space, and type II for new vessels developing above the RPE. ${ }^{45}$ Following the advent of multimodal imaging, especially SD-OCT, Freund $e^{4} \mathrm{al}^{46}$ proposed a shift in the classification of 
neovascularization towards Gass' histologic classification. They added a third entity, intraretinal neovascularization, also known as RAP, and lesions with more than one neovascular type (mixed neovascularization).

In the combined utilization of OCT/NIA study, an assumingly increased risk of GA development in CNV type II (classic) was not directly confirmed but there was a significantly higher number of eyes with type III (RAP) CNV, which developed GA. Numbers of eyes with CNV type I and CNV type III were similar, as in the CATT study.

Abdelfattah et al assessed the frequency and quantified the progression of MA in patients with $\mathrm{nAMD}$ undergoing treatment with anti-VEGF therapy for $>2$ years. ${ }^{47}$ In their final analysis they included 54 eyes of 46 patients diagnosed with wet AMD in this retrospective study. They used Cirrus SD-OCT to detect and measure GA. Patients received treatment with intravitreal ranibizumab, aflibercept, and/or bevacizumab in the study eye, and treatment was based on a treat and extend algorithm. MA was noted at baseline in 59\% of the eyes studied and progressed in all eyes over the next 2 years. MA developed by 2 years in $21 \%$ of eyes without MA at baseline. The total number of injections administered was positively correlated with GA annual enlargement rate ( $R=0.54, R^{2}=0.3, P<0.01$ ). Total number of injections not significant for the development of new GA $(R=0.26$, $\left.R^{2}=0.07, P=0.17\right)$. None of the other evaluated variables were found to predict development or progression of GA except for presence of coronary artery disease but the authors stated the study was not powered to detect small effects. The investigators concluded the rate of GA enlargement was positively correlated with the number of injections. They added however that GA did not appear to be greater than that reported for atrophy in the absence of CNV.
Subretinal drusenoid deposits and GA Other associations for GA progression and for visual loss during anti-VEGF treatment have been also investigated. A retrospective cohort study of dry AMD patients with GA, to assess the risk of progression of GA and reticular pseudodrusen (RPD), showed that presence of the latter is significantly associated with GA progression $(P<0.001),{ }^{48}$ confirming the association that Schmitz-Valkenberg et al ${ }^{49}$ had previously described.

Early detection of photoreceptor degeneration in eyes with subretinal drusenoid deposits, another term for reticular pseudodrusen, could be a biomarker prognostic of advanced AMD, including GA development with subsequent visual loss as supported mainly by the use of adaptive optics imaging ${ }^{50-53}$ in addition to older histopathologic findings of drusenoid deposits on the inner RPE in areas surrounding geographic atrophy. ${ }^{13}$

Therefore, presence of such lesions in eyes that are treated for wet AMD with anti-VEGF therapy should be noted, not only in the context of future prospective studies, but even in routine clinical practice, where a combination of continuous anti-VEGF administration and pre-existing RPD could increase chances of visual loss due to GA progression.

\section{Conclusions}

Review of the literature demonstrates that there are emerging signals of anti-VEGF treatment potentially increasing the chance of GA development and progression. The impact of GA on patients' visual function and quality of life has not been determined as studies have limited follow-up and were limited by the reliance on high-contrast, distance visual acuity as the sole functional outcome measure. High-contrast, distance visual acuity is relatively unaffected by GA until advanced stages when there is foveal involvement.

Table 1 Clinical studies outline of GA development or progression in patients treated with anti-VEGF for wet AMD

\begin{tabular}{|c|c|c|c|c|c|c|c|c|c|c|c|c|c|}
\hline \multirow[t]{2}{*}{ Study name } & \multicolumn{2}{|c|}{$\begin{array}{l}\text { Type of } \\
\text { study }\end{array}$} & \multicolumn{3}{|c|}{$\begin{array}{l}\text { Anti-VEGF } \\
\text { type }\end{array}$} & \multicolumn{2}{|c|}{ Follow-up duration } & \multicolumn{4}{|c|}{ Imaging modality type } & \multicolumn{2}{|c|}{$\begin{array}{l}\text { Most investigated and frequently identified risk } \\
\text { factors }\end{array}$} \\
\hline & $P S^{*}$ & $R s^{*}$ & $B^{*}$ & $R^{*}$ & $A^{*}$ & $\leq 1$ year & $>1$ year & $F P^{*} / F F A$ & OCT & $A F$ & $N I R$ & Treatment frequency & CNV type \\
\hline CATT (1 year) & + & - & + & + & - & + & - & + & - & - & - & + & + \\
\hline CATT (2 years) & + & - & + & + & - & - & + & + & - & - & - & + & + \\
\hline CATT (5 years) & + & - & + & + & - & - & + & + & - & - & - & - & + \\
\hline IVAN (2 years) & + & - & + & + & - & - & + & + & - & - & - & + & $\mathrm{n} / \mathrm{a}$ \\
\hline Gillies et al & - & + & + & + & + & - & + & $?$ & + & $?$ & $?$ & - & $\mathrm{n} / \mathrm{a}$ \\
\hline SEVEN-UP & - & + & - & + & - & - & + & + & + & + & - & - & $\mathrm{n} / \mathrm{a}$ \\
\hline Lois et al & - & + & - & + & - & - & + & + & - & + & + & + & + \\
\hline Young et al & - & + & + & + & - & - & + & - & + & - & - & + & + \\
\hline $\mathrm{Xu}$ et al & - & + & + & + & + & - & + & + & + & + & + & + & + \\
\hline
\end{tabular}

Abbreviations: $\mathrm{A}^{*}$, aflibercept; $\mathrm{B}^{*}$, bevacizumab; $\mathrm{FP}^{*}$, fundus photography; $\mathrm{Ps}^{*}$, prospective; $\mathrm{R}^{*}$, ranibizumab; $\mathrm{Rs}^{*}$, retrospective. 
Poor contrast sensitivity, altered dark adaptation, low luminance VA, and mesopic vision could be means of further evaluation in future studies.

The hypothesis that anti-VEGF agents are significantly associated with GA development has been supported both by animal models and studies of post-mortem human eyes.

Direct proof of a cause-effect relationship between GA and use of anti-VEGF agents in nAMD is not yet established. If an association between GA and anti-VEGF in AMD is to be directly proven in the future, it is likely that certain parameters will be implicated: type of neovascular lesion or the number of anti-VEGF injections as displayed in Table 1. Eyes with RAP and possibly eyes with classic CNV may have an increased risk of developing atrophy when treated long term with antiVEGF agents. A higher number of injections was associated with increased risk of GA development although not with an increased GA growth rate in the long term. Occult CNV has been found to be more 'resistant' to the development of GA, but also tends to cause less visual impairment. Therefore, a small amount of sub-retinal fluid (SRF) in the foveal centre displayed on the OCT, should be a reason to consider reduced treatment frequency or even just observation until further proof of CNV activity is established.

CNV development is the normal wound-healing response in an environment of chronic inflammation. Unfortunately, it ultimately leads to scar formation, which impairs central vision. ${ }^{54,55}$ Therefore, preservation of vessels and outer retinal layers from irreversible damage, rather than destruction, seems more appropriate argued $\mathrm{Kent}^{8}$ emphasizing the need to try and 'arrest' the disease at a 'pro-angiogenic stage' rather than simply targeting new vessels.

It has to be underlined that large, prospective controlled studies investigating the above would be the gold standard in addressing the association described. A multi-centre, randomized study is currently taking place to investigate non-inferiority of a treat and extend protocol of $0.5 \mathrm{mg}$ ranibizumab based on the presence of incomplete resolution of SRF $\leq 200 \mu \mathrm{m}$ at the foveal centre in patients with nAMD. ${ }^{56}$ Newly developed MA is a secondary additional endpoint and a multimodal imaging approach will be used. We are still awaiting the results of this study. However, and until further evidence is available, clinicians should aim to develop anti-VEGF treatment strategies which do not lead to over-treat whilst still maximizing treatment benefit from anti-VEGF therapy. In the future, we will need to develop alternative therapies to militate against this devastating complication, which may either represent an outcome of the natural course of the disease or be a consequence of anti-VEGF therapy or both.

\section{Conflict of interest}

The authors declare no conflict of interest.

\section{Acknowledgements}

We searched the MEDLINE/PubMed database following $\mathrm{MeSH}$ suggestions for articles including the terms 'geographic atrophy in age-related macular degeneration', 'anti vascular endothelial growth factors in the treatment of age-related macular degeneration' and 'histopathology of age-related macular degeneration'. We used the term 'long-term outcomes of anti-VEGF treatment in AMD' as a headline to locate related articles in PubMed and in order to restrict search we used the headlines 'geographic atrophy and anti-Vascular Endothelial Growth Factor agents in age related macular degeneration', 'geographic atrophy and choroidal neovascularization', and 'retinal pigment epithelial atrophy and anti-vascular endothelial growth factor treatment'. A manual search was also based on references from these articles.

\section{References}

1 Kaszubski P, Ben Ami T, Saade C, Smith RT. Geographic atrophy and choroidal neovascularization in the same eye: a review. Ophthalmic Res 2016; 55: 185-193.

2 Grunwald JE, Daniel E, Huang J, Ying GS, Maguire MG, Toth CA et al. Risk of geographic atrophy in the comparison of age-related macular degeneration treatments trials. Ophthalmology 2014; 121: 150-161.

3 Grunwald JE, Pistilli M, Ying G, Maguire MG, Daniel E, Martin DF et al. Growth of geographic atrophy in the Comparison of Age-related Macular Degeneration Treatments Trials (CATT). Ophthalmology 2015; 122: 809-816.

4 Chakravarthy U, Harding SP, Rogers CA, Downes SM, Lotery AJ, Culliford LA et al. Alternative treatments to inhibit VEGF in age-related choroidal neovascularisation: 2-year findings of the IVAN randomised controlled trial. Lancet 2013; 382: 1258-1267.

5 Seddon JM, Cote J, Page WF, Aggen SH, Neale MC. The US twin study of age-related macular degeneration: relative roles of genetic and environmental influences. Arch Ophthalmol 2005; 123: 321.

6 Hammond CJ, Webster AR, Snieder H, Bird AC, Gilbert CE, Spector TD. Genetic influence on early age-related maculopathy: a twin study. Ophthalmology 2002; 109: 730-736.

7 Miller JW. VEGF: from discovery to therapy:the champalimaud award lecture. Transl Vis Sci Technol 2016; 5: 9.

8 Kent DL. Age-related macular degeneration: beyond antiangiogenesis. Mol Vis 2014; 20: 46-55.

9 Green WR. Histopathology of age-related macular degeneration. Mol Vis 1999; 5: 27.

10 Sunness JS, Gonzalez-Baron J, Bressler NM, Hawkins B, Applegate CA. The development of choroidal neovascularization in eyes with the geographic atrophy form 
of age-related macular degeneration. Ophthalmology 1999; 106: 910-919.

11 Schatz H, McDonald HR. Atrophic macular degeneration. Rate of spread of geographic atrophy and visual loss. Ophthalmology 1989; 96: 1541-1551.

12 Green WR, Key SN 3d. Senile macular degeneration: a histopathologic study. Trans Am Ophthalmol Soc 1977; 75: 180-254.

13 Sarks JP, Sarks SH, Killingsworth MC. Evolution of geographic atrophy of the retinal pigment epithelium. Eye 1988; 2: 552-577.

14 Saint-Geniez M, Kurihara T, Sekiyama E, Maldonado AE, D'Amore PA. An essential role for RPE-derived soluble VEGF in the maintenance of the choriocapillaris. Proc Natl Acad Sci USA 2009; 106: 18751-18756.

15 Bhutto I, Lutty G. Understanding age-related macular degeneration (AMD): relationships between the photoreceptor/retinal pigment epithelium/Bruch's membrane/choriocapillaris complex. Mol Aspects Med 2012; 33: 295-317.

16 McLeod DS, Grebe R, Bhutto I, Merges C, Baba T, Lutty GA. Relationship between RPE and choriocapillaris in age-related macular degeneration. Invest Ophthalmol Vis Sci 2009; 50: 4982-4991.

17 Spaide RF, Koizumi H, Pozzoni MC. Enhanced depth imaging spectral-domain optical coherence tomography. Am J Ophthalmol 2008; 146: 496-500.

18 Kim YG, Baek SH, Moon SW, Lee HK, Kim US. Analysis of spectral domain optical coherence tomography findings in occult macular dystrophy. Acta Ophthalmol 2011; 89: e52-e56.

19 McLeod DS, Lutty GA. High resolution histologic analysis of the human choroidal vasculature. Invest Ophthalmol Vis Sci 1994; 35: 3799-3811.

20 McLeod DS, Taomoto M, Otsuji T, Green WR, Sunness JS, Lutty GA. Quantifying changes in RPE and choriocapillaris in eyes with age-related macular degeneration. Invest Ophthalmol Vis Sci 2002; 43: 1986-1993.

21 Bhutto I, McLeod DS, Jing T, Sunness JS, Seddon JM, Lutty G. Increased choroidal mast cells and their degranulation in age-related macular degeneration. Br J Ophthalmol 2016; 100: 720-726.

22 Mullins RF, Johnson MN, Faidley EA, Skeie JM, Huang J. Choriocapillaris vascular dropout related to density of drusen in human eyes with early age-related macular degeneration. Invest Ophthalmol Vis Sci 2011b; 52: 1606-1612.

23 Macular Photocoagulation Study Group. Five-year follow-up of fellow eyes of patients with age-related macular degeneration and unilateral extrafoveal choroidal neovascularization. Arch Ophthalmol 1993; 111: 1189-1199.

24 Macular Photocoagulation Study Group. Risk factors for choroidal neovascularization in the second eye of patients with juxtafoveal or subfoveal choroidal neovascularization secondary to age-related macular degeneration. Arch Ophthalmol 1997; 115: 741-747.

25 Klein R, Meuer SM, Knudtson MD, Klein BE. The epidemiology of progression of pure geographic atrophy: the Beaver Dam Eye Study. Am J Ophthalmol 2008; 146: 692-699.

26 Grob S, Luo J, Hughes G, Lee C, Zhou X, Lee J et al. Genetic analysis of simultaneous geographic atrophy and choroidal neovascularization. Eye (Lond) 2012; 26: 1106-1113.

27 Grunwald JE, Pistilli M, Daniel E, Ying G-S, Jaffe GJ, Fine S et al. Size and growth of geographic atrophy during 5 years of follow up in the Comparison of Age related Macular Degeneration Treatment Trials (CATT). ARVO 2016 Abstract No: 4292.
28 Harris MJ, Robins D, Dieter Jr JM, Fine SL, Guyton DL. Eccentric visual acuity in patients with macular disease. Ophthalmology 1985; 92: 1550-1553.

29 Gillies MC, Campain A, Barthelmes D, Simpson JM, Arnold JJ, Guymer RH et al. Long-term outcomes of treatment of neovascular age-related macular degenerationdata from an observational study. Ophthalmology 2015; 122: $1837-1845$.

30 Rofagha S, Bhisitkul RB, Boyer DS, Sadda SR, Zhang K. SEVEN-UP Study Group. Seven-year outcomes in ranibizumab-treated patients in ANCHOR, MARINA, and HORIZON: a multicenter cohort study (SEVEN-UP). Ophthalmology 2013; 120: 2292-2299.

31 Writing Committee for the UK Age-Related Macular Degeneration EMR Users Group. The neovascular agerelated macular degeneration database: multicenter study of 92976 ranibizumab injections: report 1: visual acuity. Ophthalmology 2014; 121: 1092-1101.

32 Brown DM, Kaiser PK, Michels M, Soubrane G, Heier JS, Kim RY et al. Ranibizumab versus verteporfin for neovascular age-related macular degeneration. $N$ Engl J Med 2006; 355: 1432-1444.

33 Brown DM, Michels M, Kaiser PK, Heier JS, Sy JP, Ianchulev $\mathrm{T}$ et al. Ranibizumab versus verteporfin photodynamic therapy for neovascular age-related macular degeneration: two-year results of the ANCHOR study. Ophthalmology 2009; 116: 57-65.

34 Rosenfeld PJ, Brown DM, Heier JS, Boyer DS, Kaiser PK, Chung CY et al. Ranibizumab for neovascular age-related macular degeneration. N Engl J Med 2006; 355: 1419-1431.

35 Singer MA, Awh CC, Sadda S, Freeman WR, Antoszyk AN, Wong P et al. HORIZON: an openlabel extension trial of ranibizumab for choroidal neovascularization secondary to age-related macular degeneration. Ophthalmology 2012; 119: 1175-1183.

36 Bhisitkul RB, Desai SJ, Boyer DS, Sadda SVR, Zhang K. Fellow eye comparisons for 7-year outcomes in ranibizumab-treated AMD subjects from ANCHOR, MARINA, and HORIZON (SEVEN-UP Study). Ophthalmology 2016; 123: 1269-1277.

37 Sadda S, Tuomi L, Ding B, Hopkins JJ. Development of Atrophy in Neovascular AMD Treated with Anti-VEGF Therapy: Results of the HARBOR Study. Paper Presented at AAO Annual Meeting, Retina Subspecialty Day; 17 October 2014; Chicago, IL, USA.

38 Lois N, McBain V, Abdelkader E, Scott NW, Kumari R. Retinal pigment epithelial atrophy in patients with exudative age-related macular degeneration undergoing anti-vascular endothelial growth factor therapy. Retina 2013; 33: 13-22.

39 Young M, Chui L, Fallah N, Or C, Merkur AB, Kirker AW et al. Exacerbation of choroidal and retinal pigment epithelial atrophy after anti-vascular endothelial growth factor treatment in neovascular age-related macular degeneration. Retina 2014; 34: 1308-1315.

40 Xu L, Mrejen S, Jung JJ, Gallego-Pinazo R, Thompson D, Marsiglia $\mathrm{M}$ et al. Geographic atrophy in patients receiving anti-vascular endothelial growth factor for neovascular agerelated macular degeneration. Retina 2015; 35: 176-186.

41 Engelbert M, Zweifel SA, Freund KB. Long-term follow-up for type 1 (subretinal pigment epithelium) neovascularization using a modified 'treat and extend' dosing regimen of intravitreal antivascular endothelial growth factor therapy. Retina 2010; 30: 1368-1375. 
42 Grossniklaus HE, Green WR. Choroidal neovascularization. Am J Ophthalmol 2004; 137: 496-503.

43 Macular Photocoagulation Study Group. Laser photocoagulation of subfoveal neovascular lesion in agerelated macular degeneration: results of a randomized clinical trial. Arch Ophthalmol 1991; 109: 1220-1231.

44 Macular Photocoagulation Study Group. Occult choroidal neovascularization. Influence on visual outcome in patients with age-related macular degeneration. Arch Ophthalmol 1996; 114: 400-412; Erratum in Arch Ophthalmol 1996; 114: 1023.

45 Gass JD. Biomicroscopic and histopathologic considerations regarding the feasibility of surgical excision of subfoveal neovascular membranes. Am J Ophthalmol 1994; 118: 258-298.

46 Freund K, Bailey MD, Zweifel SA, Engelbert M. Do we need a new classification for choroidal neovascularization in agerelated macular degeneration? Retina 2010; 30: 1333-1349.

47 Abdelfattah NS, Zhang H, Boyer DS, Sadda SR. Progression of macular atrophy in patients with age-related macular degeneration undergoing antivascular endothelial growth factor therapy. Retina 2016; 36: 1843-1850.

48 Marsiglia M, Boddu S, Chen CY, Jung JJ, Mrejen S, GallegoPinazo R et al. Correlation between neovascular lesion type and clinical characteristics of nonneovascular fellow eyes in patients with unilateral, neovascular age-related macular degeneration. Retina 2015; 35: 966-974.

49 Schmitz-Valckenberg S, Steinberg JS, Fleckenstein M, Visvalingam S, Brinkmann CK, Holz FG. Combined confocal scanning laser ophthalmoscopy and spectral-domain optical coherence tomography imaging of reticular drusen associated with age-related macular degeneration. Ophthalmology 2010; 117: 1169-1176.

50 Mrejen S, Sato T, Curcio CA, Spaide RF. Assessing the cone photoreceptor mosaic in eyes with pseudodrusen and soft drusen in vivo using adaptive optics imaging. Ophthalmology 2014; 121: 545-555.

51 Zweifel SA, Imamura Y, Spaide TC, Fujiwara T, Spaide RF. Prevalence and significance of subretinal drusenoid deposits (reticular pseudodrusen) in age-related macular degeneration. Ophthalmology 2010; 117: 1775-1781.

52 Zweifel SA, Spaide RF, Curcio CA, Malek G, Imamura Y. Reticular pseudodrusen are subretinal drusenoid deposits. Ophthalmology 2010; 117: 303-312.

53 Spaide RF, Curcio CA. Drusen characterization with multimodal imaging. Retina 2010; 30: 1441-1454.

54 Green WR, Enger C. Age-related macular degeneration histopathologic studies. The 1992 Lorenz E. Zimmerman Lecture. Ophthalmology 1993; 100: 1519-1535.

55 Kent DL, Sheridan C. Choroidal neovascularization: a wound healing perspective. Mol Vis 2003; 9: 747-755.

56 Arnold J, Markey CM, Kurstjens NP, Guymer RH. The role of sub-retinal fluid in determining treatment outcomes in patients with neovascular age-related macular degeneration - a phase IV randomised clinical trial with ranibizumab: the FLUID study. BMC Ophthalmology 2016; 16: 31. 